\title{
¿Cómo enseñar una segunda lengua en el aula? Beneficios asociados al enfoque AICLE
}

\author{
How toTeach a Second Language in the Classroom? Benefits Associated with the CLIL \\ Approach
}

\section{¿Como ensinar uma segunda língua na sala de aula? Benefícios associados à abordagem AICLE}

María José Bolarín-Martínez

Universidad de Murcia

Murcia, España

mbolarin@um.es

https://orcid.org/0000-0002-9716-8584

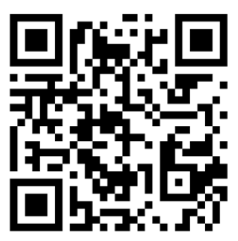

Mónica Porto-Currás

Universidad de Murcia

Murcia, España

monicapc@um.es

https://orcid.org/0000-0002-4938-0572

María Lova-Mellado

Centro de Educación Infantil y Primaria San Francisco

Murcia, España

maria88_trebol@hotmail.com

https://orcid.org/0000-0001-5456-9567

Recibido • Received • Recebido: 30 / 10 / 2019

Corregido • Revised • Revisado: 11 / 12 / 2020

Aceptado • Accepted • Aprovado: 14 / 03 / 2021

\begin{abstract}
Resumen:
Introducción. El presente artículo expone cómo el enfoque de aprendizaje integrado de contenidos y lenguas extranjeras (AICLE) favorece la construcción de un aprendizaje bilingüe, que aporta diferentes beneficios al alumnado. Objetivo. El objetivo de este trabajo reside en valorar si los cambios conceptuales, metodológicos y culturales que aporta la enseñanza de una segunda lengua mediante el enfoque AICLE conllevan, no solo una mejora del aprendizaje que realiza el alumnado, sino también una revisión y renovación de la práctica docente del personal implicado. Análisis. Con este fin, el artículo presenta una aproximación a la conceptualización del enfoque AICLE y un análisis detallado de las estrategias metodológicas que el profesorado debe emplear para facilitar la implementación de este enfoque en el aula. Conclusiones. La revisión de estas estrategias metodológicas permite resultados, conclusiones que para desarrollarlas en la práctica es necesario que el personal docente efectúe una reflexión profunda sobre la enseñanza que está realizando y los objetivos que pretende alcanzar. Si esta reflexión se genera y generaliza entre todo el profesorado
\end{abstract}


http://doi.org/10.15359/ree.25-2.26

http://www.una.ac.cr/educare

educare@una.ac.cr

participante en programas de enseñanza bilingües, se estarán consiguiendo muchos de los retos pedagógicos que desde hace décadas se reclaman a la educación obligatoria, y así, se permitiría tanto la renovación de la enseñanza de las lenguas como del sistema educativo en general.

Palabras claves: Aprendizaje integrado de contenidos y lenguas extranjeras; programas bilingües, metodología AICLE; estrategias; formación docente.

\begin{abstract}
:
Introduction. This article discusses how the Content and Languages Integrated Learning (CLIL) favors the construction of bilingual learning that brings different benefits to students. Aims. This research aims to assess whether the conceptual, methodological, and cultural changes, brought by the teaching of a second language through the CLIL approach, involve not only an improvement in the learning done by students but also a review and renewal of the teaching practice of the teachers involved. Analysis. To accomplish this aim, the article considers the CLIL approach's conceptualization and a detailed analysis of the methodological strategies that teachers should employ to facilitate the implementation of this approach in the classroom. Conclusions. The review of these methodological strategies leads to results and conclusions that, in order to develop them in practice, teachers have to reflect deeply on the teaching they are doing and the objectives they intend to achieve. If this reflection is generated and generalized among all the teachers participating in Bilingual Teaching Programs, many of the pedagogical challenges claimed for compulsory education for decades will be being achieved, allowing both the renewal of language teaching and the system educational in general.
\end{abstract}

Keywords: Content and language integrated learning; methodology CLIL; strategies; teacher education.

\title{
Resumo:
}

Introdução. O presente artigo explica como a abordagem da Aprendizagem Integrada de Conteúdo e Línguas Estrangeiras (AICLE) promove a construção da aprendizagem bilíngue, que traz diferentes benefícios para o grupo de estudantes. Objetivo. O objetivo da discussão deste trabalho consiste em avaliar se as mudanças conceituais, metodológicas e culturais oferecidas pelo ensino de uma segunda língua através da abordagem AICLE conduzem não só a uma melhoria na aprendizagem do grupo de estudantes, más também uma revisão e renovação da prática docente dos professores envolvidos. Análise. Dessa forma, o artigo aproxima-se a conceituação da abordagem AICLE e, além disso, explica cuidadosamente as estratégias metodológicas que os professores devem utilizar para facilitar a implementação dessa abordagem em sala de aula. Conclusão. A revisão dessas estratégias metodológicas permite resultados, concluindo que, para desenvolvê-los na prática, é necessário que os professores façam uma reflexão profunda sobre sua prática pedagógica e os objetivos que visam alcançar. Se esta reflexão é gerada e generalizada entre todos os professores participantes de Programas de Ensino Bilíngues, muitos dos desafios pedagógicos que têm sido chamados para a educação obrigatória por décadas serão alcançados, permitindo tanto a renovação no ensino de línguas, como do sistema educativo em geral.

Palavras-chave: Aprendizagem integrada de conteúdo e línguas estrangeiras; programas bilíngues; metodologia AICLE, estratégias, formação docente.

María José Bolarín-Martínez, Mónica Porto-Currás y María Lova-Mellado

Los artículos de la Revista Electrónica Educare del Centro de Investigación y Docencia en Educación de la Universidad Nacional, Costa Rica, se comparten bajo términos de la Licencia Creative Commons: Reconocimiento, No Comercial, Sin Obra Derivada 3.0 Costa Rica. Las autorizaciones adicionales a las aquí delimitadas se pueden obtener en el correo: educare@una.cr 


\section{Introducción}

La sociedad actual apuesta por una globalización, por un acercamiento de los pueblos. Si bien dicha globalización se debe plantear desde diferentes facetas sociales, no cabe duda de que una de ellas es la educación. En este sentido, conocer una segunda lengua (L2) permite abrir fronteras, comprender otros contextos, acercar culturas, progresar y jugar un papel decisivo en el desarrollo de un país; ser bilingüe es un requisito indispensable para responder a las exigencias de una sociedad abierta y globalizada. En esta línea, se manifiesta la necesidad de fomentar, desde la enseñanza obligatoria, una sociedad conocedora de varias lenguas que permita a sus estudiantes transitar, estudiar y desenvolverse en una sociedad plural, competitiva abierta y en continua evolución (Roza González, 2005). Desde esta premisa, la introducción en los currículos escolares del conocimiento de una segunda lengua contribuye a conocer otros contextos, aunar culturas y al desarrollo de un país.

Dejando a un lado bondades de diferente naturaleza (económicas, políticas, socioculturales, etc.), el bilingüismo conlleva numerosos beneficios para el alumnado y para el resto de la comunidad educativa, que se presentan de manera resumida a continuación.

En primer lugar, mejora la competencia lingüística de la lengua materna. El estudiantado realiza grandes progresos en su lengua materna al enfrentarse a estructuras de la segunda lengua. Se produce así una transferencia positiva, el aprendizaje de dos idiomas lejos de afectarse negativamente, se apoya mutuamente (Dobson et al., 2011). En este sentido, el aprendizaje del vocabulario de una segunda lengua favorece el incremento y adquisición del significado de nuevas palabras en la lengua materna, y contribuye a mejorar la comprensión de la lengua materna. Más concretamente, la comprensión de la relación simbólica existente entre las palabras escritas y su significado (Signoret Dorcasberro, 2003).

En segundo lugar, favorece el aprendizaje denuevas estrategias cognitivas. "El [estudiantado] bilingüe puede utilizar dos [niveles] diferentes de razonamiento lógico ... y un sistema de clasificación extendido" (Ardila, 2012, p. 102), y es capaz de dirigir la atención selectivamente e inhibirla ante información irrelevante, pero más compleja (Signoret Dorcasberro, 2003). En consecuencia, demanda un desarrollo de la atención y favorece la creatividad y flexibilidad cognitiva (Cummins, 2002), al obligarle a usar los recursos que posee en los dos idiomas. De esta forma, ayuda a desarrollar un número mayor de estrategias de comunicación y de resolución de problemas (Cummins, 2002; Döpke, 2003; Lorenzo et al., 2011; Signoret Dorcasberro, 2003), al emplear los recursos en las dos lenguas, además permite poseer un mayor desarrollo y uso de estrategias de comunicación y resolución de problemas (Bialystok et al., 2010; Cummins, 2002), pues ante un conflicto lingüístico es capaz de adaptarse al contexto y controlar mejor esta nueva situación. 
http://doi.org/10.15359/ree.25-2.26

http://www.una.ac.cr/educare

educare@una.ac.cr

En tercer lugar, son conocidos los estudios que prueban que mejora resultados académicos (Dobson et al., 2011) y la actitud positiva hacia el aprendizaje en general (Aragón-Méndez, 2007).

Y, en cuarto lugar, desarrolla la competencia cultural. Está demostrado que aprender una o varias nuevas lenguas y dominarlas para desenvolverse con naturalidad en situaciones cotidianas implica la transferencia de la cultura que sustenta, con los valores, intereses y visiones del mundo propios de esta (Pavón Vázquez y Rubio, 2010; Pérez Márquez, 2008; Pérez Vidal, 2008).

Desdeesta perspectiva, esteartículo tieneel propósito de presentarelenfoque denominado aprendizaje integrado de contenidos y lenguas extranjeras (AICLE) o de contenidos e idiomas que se han ido generalizando en toda Europa, con el fin de valorar si los cambios conceptuales, metodológicos y culturales que este enfoque conlleva suponen una revisión y renovación de la práctica docente del personal implicado (Custodio Espinar, 2019). El informe (EURYDICE y Comisión Europea, 2006) constató que hoy es uno de los enfoques más ampliamente conocidos y con mayor envergadura en el ámbito educativo en el aprendizaje de una segunda lengua.

\section{Enfoque aprendizaje integrado de contenidos y lenguas extranjeras}

Este enfoque surge en especial, pero no únicamente, como una estrategia para el logro de habilidades lingüísticas y aprendizaje de las lenguas extranjeras. La propia delimitación del término AICLE entraña dificultades reflejadas sobre todo en el debate existente acerca de su definición como enfoque, como metodología, como modelo o como estrategia. La definición más completa la aportan Marsh et al. (2010), que lo consideran como un enfoque educativo con una clara finalidad: utilizar la segunda lengua para enseñar $y$, por tanto, para promover el dominio del contenido y el lenguaje a la vez. Por ello, la integración de contenidos académicos y lengua facilita que se conciba como un enfoque exitoso, si el estudiantado aprende tanto los contenidos académicos de las áreas enseñadas en la segunda lengua como la lengua en sí misma. De esta naturaleza dual se infiere que el aprendizaje paralelo de contenidos y lenguas no solo afecta al qué sino también al cómo: desde esta perspectiva se requiere un cambio sustancial en la metodología, que debe considerar la implementación de nuevas habilidades y estrategias de enseñanza.

\section{Implementación del enfoque: Metodología de enseñanza}

El lenguaje es el instrumento empleado para la comprensión y adquisición de nuevos contenidos académicos por parte del estudiantado, es el medio para el intercambio de ideas acerca de los conocimientos aprendidos entre el alumnado y el alumnado-profesorado. Por ello, la metodología adquiere gran relevancia en este enfoque y el profesorado implicado necesita salir de 
su zona de confort y cambiar los métodos y estrategias empleadas para enseñar los contenidos en una segunda lengua. En consecuencia, una implementación con éxito reclama: una planificación fundamentada, la integración de ambas lenguas y estrategias metodológicas adecuadas.

\section{Planificación}

Al profesorado le corresponde la elaboración del currículo integrado de las lenguas junto al profesorado de áreas no lingüísticas. Para ello, se deben tener en cuenta los elementos en el marco de las 4Cs (Coyle et al., 2010), que suponen el marco conceptual empleado en la planificación y diseño de actividades y tareas. Se considera una herramienta de planificación de gran utilidad al integrar las cuatro dimensiones claves: contenido, comunicación, cognición y cultura.

a) Contenido: hace referencia a los contenidos académicos de las diferentes áreas enseñadas a través de la segunda lengua. Es el eje de la enseñanza bilingüe, el punto inicial del proceso de planificación de los conocimientos, determinando así el aprendizaje de contenidos y habilidades, proporciona oportunidades para estudiar el contenido mediante diversas perspectivas que favorecen una mejor y más profunda comprensión de estos mismos. Asimismo, permite entender la terminología específica de las áreas en la segunda lengua y prepara al alumnado para estudios futuros y para su vida laboral.

b) Comunicación o lenguaje: alude a la lengua que el alumnado va a necesitar para comprender los contenidos académicos, para participar en las actividades de aprendizaje e interaccionar con sus compañeros y compañeras sobre los aprendizajes. El lenguaje ha de ser claro y comprensible, con el fin de facilitar al estudiantado la adquisición de los nuevos conocimientos. Se debe considerar el lenguaje del aprendizaje, el lenguaje para el aprendizaje y el lenguaje a través del aprendizaje. Se trata de planificar qué vocabulario y frases concretas el alumnado necesitará a lo largo de cada unidad didáctica, qué tipo de lenguaje va a ser preciso para la producción oral y para las actividades en parejas o en grupo como debates, así como las estructuras y expresiones lingüísticas determinadas que usarán.

c) Cognición: está ligada al contenido, pues se trata de activar procesos y habilidades cognitivas complejas promoviendo un aprendizaje más exitoso. Para ello, trata de animar al estudiantado a pensar con el fin de que sean capaces de entender y crear sus propias interpretaciones de los conocimientos aprendidos. Coyle (2002) recomienda usar los seis procesos cognitivos (conocimiento, comprensión, aplicación, análisis, síntesis y evaluación) para fomentar habilidades de pensamientos superiores o complejas. 
http://doi.org/10.15359/ree.25-2.26

http://www.una.ac.cr/educare

educare@una.ac.cr

d) Cultura: favorece la construcción de conocimiento y comprensión intercultural, puede fomentar el desarrollo de habilidades de comunicación intercultural y el aprendizaje sobre diferentes países o regiones, así como introducir al estudiantado en un contexto cultural más amplio. Se refiere, por tanto, a la relación existente entre la lengua y la cultura.

\section{Integración de ambas lenguas}

El estudiantado debe aprender el discurso relativo a los conocimientos de las áreas impartidas en la segunda lengua, se emplea la segunda lengua para aprender las áreas a la vez que se aprende a usar esa lengua. Por ello, se contribuye al dominio de ambas habilidades comunicativas, donde la estructura de contenidos es la que va a marcar la secuenciación lingüística, de forma que se aborda la gramática conforme los conocimientos lo requieren. Por ello, el personal docente debe meditar y planificar las estrategias necesarias para practicar y fomentar la segunda lengua.

\section{Estrategias metodológicas}

Las estrategias deben facilitar la comprensión de los contenidos académicos al estudiantado y promover un uso activo de la lengua. Se exponen las principales a desarrollar:

\section{Uso de ambas lenguas con fines comunicativos}

Desde este enfoque se deben ofrecer oportunidades para que el estudiantado aprenda la segunda lengua de manera natural e innovadora, promoviendo el uso de actividades y tareas que potencien la comunicación real (Dalton-Puffer, 2008; Maljers et al., 2007; Wolff, 2012). Para ello, el profesorado debe proporcionar un entorno favorecedor que fomente la motivación del alumnado por el uso de esta lengua, de lo contrario, se limitarán a utilizar siempre la lengua materna. Kiely (2011) expresa algunos principios clave relativos al uso de las lenguas: maximizar la exposición y uso de la segunda lengua en las clases bilingües; comprobar la comprensión utilizando la lengua materna; enseñar los términos de las áreas impartidas a través de la segunda lengua en la lengua materna y usar la lengua materna para apoyar el aprendizaje. En definitiva, la idea que parece subsistir acerca del empleo de la lengua materna y la segunda lengua en los contextos escolares es la flexibilidad en el empleo de ambas.

\section{Uso de las metodologías activas}

Este enfoque exige un cambio de paradigma de enseñanza, centrado en un traspaso del protagonismo entre los roles profesor-estudiantes y la máxima calidad didáctica: "La metodología de la enseñanza de contenidos cambia para hacerse más activa y centrada en el 
alumno" (Halbach, 2015, p. 18). El cambio en la enseñanza de la lengua conlleva cambios en la metodología (Coyle, 2002): implica la redefinición de metodologías que tengan en cuentan el lenguaje del personal docente y alumnado y que, consecuentemente, deriva en una mayor participación e interacción en las lecciones. El diálogo e intercambio de ideas entre escolares juega un rol fundamental en estos programas, relega a un segundo lugar la tradicional interacción docente-estudiante. El personal docente ha de moverse de la interacción docenteestudiante a la interacción estudiante-estudiante (Guazzieri, 2008). Este enfoque involucra al alumnado en procesos cognitivos exigentes, ya que requiere un esfuerzo extra de este por comprender los contenidos académicos, decodificar la información trasmitida en una lengua diferente a la materna y comunicar sus conocimientos acerca de lo aprendido (Pavón Vázquez y Ellison, 2013). La enseñanza supone una mayor implicación del alumnado haciéndolo sentir partícipe imprescindible en los procesos de enseñanza-aprendizaje, donde la interacción social cobra un papel relevante (Guazzieri, 2008; Pastor Martínez, 2011).

\section{Empleo de estrategias de andamiaje}

El esfuerzo cognitivo del estudiantado al comprender y adquirir contenidos académicos en una lengua diferente a la materna requiere que el personal docente le proporcione oportunidades facilitadoras del aprendizaje de los conocimientos y de la segunda lengua. Por ello, es imprescindible que el profesorado conozca y desarrolle diversas estrategias de enseñanza, una de las más importantes es las "estrategias de andamiaje" (de Matos, 2014; Pavón Vázquez y Ellison, 2013). A partir de la propuesta de Applebee (1986, citado por Delmastro, 2008), se proponen los siguientes criterios a tener en cuenta por el personal docente para lograr un efectivo andamiaje:

- Crear un ambiente estructurado y organizado que propicie el desarrollo natural del pensamiento y de lenguaje del alumnado. En definitiva, se trata de crear las condiciones que lo motiven a aprender.

- Las propuestas o tareas deben estar centradas en el alumnado y, por ello, combinar niveles de dificultad, con posibilidades de realización. Es decir, tareas que le supongan un reto y un aprendizaje, a la vez que se adapten a sus posibilidades de realización, teniendo en cuenta su grado de madurez.

- El progreso del alumnado implica una mayor responsabilidad e interacción sobre el desarrollo de la tarea y un aumento en su nivel de competencia.

- Asumir la responsabilidad compartida, las tareas se realizan a través de la interacción, en este sentido, el maestro o la maestra colabora en el aprendizaje del alumnado. 
http://doi.org/10.15359/ree.25-2.26

http://www.una.ac.cr/educare

educare@una.ac.cr

En definitiva, se trata de "proporcionar al alumnado apoyos o herramientas auxiliares, andamiajes [para resolver] ... la tarea con el apoyo del profesor o trabajando con otros compañeros, en su zona de desarrollo próximo (donde es posible el aprendizaje con ayuda) y una vez asentado el conocimiento, será capaz de completar la misma tarea de forma individual sin ayuda" (Bolarín Martínez et al., 2012, p. 264). En esta línea, Massler et al. (2011) proponen una clasificación de estrategias de andamiaje que distribuyen en 3 grupos: verbales, de contenido y de procesos de enseñanza aprendizaje (Tabla 1).

Tabla 1: Estrategias de andamiaje

\begin{tabular}{ll}
\hline A. Técnicas de andamiaje verbal & $\begin{array}{l}\text { - Uso adecuado del lenguaje } \\
\text { - Lenguaje de animación } \\
\text { - Repetición en las lecciones } \\
\text { - Modelo del uso correcto del lenguaje } \\
\text { - Uso de la lengua materna }\end{array}$ \\
\hline B. Técnicas de andamiaje de contenidos & $\begin{array}{l}\text { - Seleccionar y adaptar al nivel del estudiantado } \\
\text { - Partir de conocimientos y experiencias previas } \\
\text { - Definir y revisar contenidos y objetivos con el alumnado }\end{array}$ \\
\hline $\begin{array}{l}\text { C. Técnicas de andamiaje de procesos de } \\
\text { enseñanza-aprendizaje }\end{array}$ & - Potenciar el uso de diversas estrategias de aprendizaje \\
\hline
\end{tabular}

Nota: Elaboración propia.

\section{A. Técnicas de andamiaje verbal}

Estas técnicas pueden estar focalizadas en hacer accesible al estudiantado el lenguaje empleado por su docente y orientadas a las ayudas que se le pueden suministrar para que sea capaz de expresar su comprensión de los contenidos y, a su vez, participar de manera activa en las clases a pesar de sus diferentes niveles de competencia lingüística.

Las técnicas que tienen como objetivo hacer accesible al estudiantado el lenguaje, aluden a los cambios que el personal docente realiza en su lenguaje con el fin de lograr ser entendidos por el estudiantado. Y se pueden distinguir entre:

a) Uso adecuado del lenguaje: El personal docente está tan preocupado por las exigentes capacidades cognitivas que la integración de la lengua y el contenido demanda en el estudiantado que tiende a simplificar demasiado su lenguaje, a usar solo frases muy cortas y sencillas y a repetir en diversas ocasiones las mismas estructuras. Teniendo en cuenta que uno de los requisitos esenciales para un aprendizaje efectivo es proporcionar una rica exposición a la segunda lengua, la simplificación del lenguaje no parece ser una de las mejores opciones. Por ello, es recomendable modificar el lenguaje, hacer el lenguaje más accesible al estudiantado moderando la velocidad del discurso y enfatizando la entonación de palabras claves (Pavón Vázquez y Ellison, 2013). 
b) Lenguaje de animación: un enfoque multimodal, la combinación del lenguaje verbal y no verbal es una de las estrategias más conocidas y empleadas por el personal docente. Los gestos, mímica, expresiones faciales, recursos visuales, son esenciales. Por tanto, es obvio que acompañar el discurso docente con estos recursos facilita la comprensión y favorece un mayor éxito en el aprendizaje de los contenidos académicos.

c) Repetición en las lecciones: el uso de repeticiones, sinónimos y antónimos y parafraseo ayuda al alumnado a entender mejor la información transmitida por su profesorado.

d) Docentes como modelos de uso correcto del lenguaje: el profesorado puede ser el único modelo lingüístico accesible para sus estudiantes. Por ello, el personal docente puede repetir o parafrasear las respuestas del alumnado de manera adecuada con el fin de ir corrigiendo su uso de la segunda lengua y lograr pertinentes habilidades lingüísticas (Massler et al., 2011). El énfasis en entornos AICLE reside en la comunicación frente a la norma. La capacidad para comunicarse con los demás empleando la segunda lengua adquiere más relevancia que la corrección en esa lengua. La fluidez debe primar frente a la exactitud. No obstante, aunque la atención en las clases se focalice en la comunicación del estudiantado, es necesario encontrar momentos en los que se le modele sus discursos, ofreciéndole una pronunciación adecuada, con el fin de asegurar una comunicación efectiva. Consecuentemente, es esencial proporcionar vocablos correctos que contribuyan al aprendizaje de esa comunicación adecuada desde sus inicios en la adquisición de una segunda lengua y al logro, con el transcurso del tiempo, de una precisión en sus habilidades lingüísticas.

e) Uso de la lengua maternal: el rol concedido a las lenguas en la enseñanza bilingüe ha ocasionado distinciones en la implementación de los programas bilingües. Los programas fundamentados en el enfoque AICLE promueven un bilingüismo aditivo, en el que tanto la lengua materna como la segunda lengua adquieren gran relevancia. Lázaro y García Mayo (2012) y Méndez García y Pavón Vázquez (2012) resaltan el papel que posee la lengua materna como herramienta de aprendizaje. Ambas son lenguas vehiculares propiciando comunicación, enseñanza, aprendizaje y cultura.

\section{B. Técnicas de andamiaje de contenidos}

Estas técnicas permiten apoyar la comprensión de los contenidos académicos al estudiantado, de los conceptos, así como de las tareas. Para ello, el profesorado debe:

a) Seleccionar y adaptar el conocimiento del contenido al nivel de desarrollo y cognitivo del estudiantado: el profesorado ha de utilizar técnicas con el fin de adaptar su discurso al estudiantado y favorecer el aprendizaje tanto de la segunda lengua como de la comprensión de los conocimientos. 
http://doi.org/10.15359/ree.25-2.26

http://www.una.ac.cr/educare

educare@una.ac.cr

b) Partir de conocimientos y experiencias previas, vinculando los intereses y la vida del estudiantado: instar al estudiantado a reflexionar sobre lo que ya saben acerca de una temática determinada y preguntarles lo que les gustaría saber sobre la esta misma al inicio de la unidad didáctica permite relacionar los conocimientos con sus intereses e inquietudes y facilita la comprensión del alumnado.

c) Definir, mostrar y revisar contenido y objetivos de lenguaje con el estudiantado: expresar los conocimientos que el alumnado posee sobre un tema y lo que les gustaría conocer sobre este al inicio de la unidad didáctica facilita hacerlos conocedores de los objetivos que se pretenden lograr al finalizar las explicaciones y tareas sobre el mismo. Asimismo, revisar profesores y alumnos lo mencionado al inicio de la unidad al concluir la misma, permite que los escolares sean conocedores de su aprendizaje.

De manera más concreta, las técnicas que promueven la comprensión de los conceptos son las siguientes: uso de técnicas de visualización, mediante el uso de imágenes y objetos de su entorno cercano que le son familiares al estudiantado, la comprensión de conceptos; descubrimiento activo de conceptos, mediante actividades manipulativas y prácticas que le permitan explorar los nuevos conocimientos (Massler et al., 2011). Se trata de que el alumnado "aprenda haciendo", participe experimentando y manipulando, descubriendo por sí mismo los nuevos conocimientos; de proporcionar revisión de vocabulario clave y conceptos clave de contenido durante las lecciones; de verificar regularmente la comprensión, dar retroalimentación y comprobar la comprensión de los contenidos académicos, y dar una retroalimentación sobre sus aprendizajes.

\section{Técnicas de andamiaje de procesos de enseñanza aprendizaje}

Estas técnicas apoyan los procesos de aprendizaje del estudiantado, al fomentar el uso de diversas estrategias de aprendizaje para facilitar la adquisición de conocimientos. Algunas de ellas son: lectura de textos, uso de técnicas de escaneo para leer un texto, enseñanza de contenido de estrategias de trabajo específicas (encuestas), enseñanza con la ayuda de organizadores avanzados o gráficos (líneas de tiempo, diagramas de flujo, mapas semánticos, etc.) y el uso de mnemotecnia (Massler et al., 2011) que al favorecer la memorización de algún contenido promueven su asimilación.

Aunque resulta difícil explicar y diferenciar en la implementación de este enfoque integrado las diferentes estrategias, pasamos a continuación a expresar brevemente algunas orientaciones de cómo se pueden implementar y materializar en actividades de aula.

En primer lugar, destacar que las estrategias presentadas para facilitar la comprensión de los contenidos académicos y la integración de contenidos en la lengua materna y la segunda se basan en la coordinación entre el profesorado para impartir los mismos contenidos en las dos lenguas diferentes, de manera que el estudiantado asimila los contenidos en las dos lenguas de manera paralela e integrada. 
A partir de esta coordinación, el profesorado selecciona los contenidos lingüísticos que son necesarios para que el alumnado comprenda y exprese los conceptos clave para trabajar en la unidad de programación propuesta, atendiendo su nivel y sus conocimientos previos (técnicas de andamiaje de contenidos). A partir de las ideas o conocimientos previos y, mediante la propuesta de metodologías activas como debates, grupos de discusión sobre lecturas (técnicas de andamiaje de procesos de enseñanza aprendizaje) y videos en la lengua extranjera, el alumnado debe manifestar su dominio conceptual (técnicas de andamiaje de contenidos) a la vez que su dominio en la adquisición de las estructuras lingüísticas de la segunda lengua (técnicas de andamiaje de verbal-uso de lengua con fines comunicativos).

El empleo del uso de variedad de estrategias de enseñanza de manera integrada juega un rol esencial en estos entornos educativos.

\section{Conclusiones}

El bilingüismo en las aulas no es solo cambiar la lengua de enseñanza, este enfoque es mucho más que el incremento de la exposición a esa lengua haciendo lo mismo que en la lengua materna. Un modelo basado en la transmisión de conocimientos docente-estudiante, en la simple traducción de actividades de lengua materna a la segunda lengua es inviable para el desarrollo de la competencia comunicativa que demanda la sociedad actual y para la adquisición de conocimientos. Para garantizar el éxito y la calidad de la implementación de este enfoque, el profesorado debe reflexionar y cuestionar sus formas de trabajar en el aula, un cambio de código de la lengua no es suficiente para la implementación exitosa de la enseñanza en una segunda lengua. Todo ello implica un cambio en la manera de afrontar la tarea educativa en las aulas, el uso de diversas estrategias de enseñanza por parte del personal docente para facilitar la comprensión de los contenidos y garantizar un aprendizaje relevante.

Además, la adquisición de una segunda lengua puede ser la forma más directa "para entender que ... [la] lengua [materna] representa ... una forma específica y particular de organizar la realidad y [las] experiencias personales, no la única forma de conceptualizar el mundo" (Ardila, 2012, p. 103). Por ello, la enseñanza de una segunda lengua en las aulas debe ser un reto de las diferentes políticas para fomentar las relaciones sociales y la comunicación entre los diferentes pueblos y, en este punto, es donde tienen cabida los programas bilingües como opción para poseer un buen dominio de una segunda lengua. Dichos programas tienen como finalidad que el alumnado aprenda un segundo idioma sin olvidar el suyo propio; así, los idiomas se emplean como lenguas vehiculares a través de las cuales el estudiantado logra los contenidos lingüísticos y académicos concretados en el currículo y la competencia comunicativa para manejarse de manera espontánea en situaciones de la vida cotidiana, otorgándole así una aplicación práctica (Cummins, 2002). Se destacan los beneficios cognitivos que la enseñanza de una segunda lengua posee en el alumnado y, en consecuencia, en su autonomía y en un mayor grado de madurez (Frigols Martín y Marsh, 2014). Por tanto, se puede afirmar que el 
http://doi.org/10.15359/ree.25-2.26

http://www.una.ac.cr/educare

educare@una.ac.cr

bilingüismo facilita el aprendizaje de una segunda lengua y contribuye al desarrollo de la competencia cultural y un sentimiento de ciudadanía más global, e integra la concienciación y la comprensión intercultural en el currículo (Coyle, 2002; Ramos, 2011).

Como ya se expresó en un trabajo anterior (Bolarin et al., 2012), todo ello demanda la formación del profesorado para dar respuesta a tales necesidades, que debe incluir tanto aspectos teóricos como prácticos. Se requiere un conocimiento profundo de los elementos implicados en el aprendizaje de la lengua extranjera, del que dependen las metodologías que puedan favorecer y mejorar el aprendizaje del estudiantado, puesto que sin ella se puede ver perjudicada la implantación de los programas bilingües (Bovellan, 2014). Y también la reflexión docente desempeña, en el cambio, un rol fundamental. La autorreflexión adquiere gran relevancia en el ámbito docente al contribuir a que los educadores y educadoras mediten sobre su enseñanza y justifiquen las decisiones de estas mismas (Harjanne y Tella, 2008). El profesorado tiene que ser consciente de sus prácticas educativas y reflexionar sobre ellas, con el fin de mejorarlas. La reflexión permite llevar la teoría a la práctica, meditar sobre la labor que realizamos día tras día, cuestionarla y, a su vez, cambiar la práctica docente y mejorarla.

En definitiva, los cambios introducidos en la implementación del enfoque AICLE por el personal docente van más allá de las áreas impartidas en otra lengua, también abarcan las áreas que se imparten en lengua materna. Las competencias y estrategias de enseñanza empleadas en la enseñanza de la segunda lengua son transferidas a la enseñanza en la lengua materna. Por ello, se puede mencionar que, bien entendido, el enfoque AICLE contribuye a una mejora de la práctica docente y a una nueva visión sobre la enseñanza y aprendizaje, por lo que no se puede considerar un enfoque exclusivo de las áreas lingüísticas.

Sin duda, el uso por parte del profesorado de las estrategias planteadas como esenciales en el enfoque AICLE, tal como se han expuesto a lo largo de este artículo, se pueden considerar como premisas didácticas básicas para fomentar un proceso de enseñanza de calidad. Así, una planificación detallada en la que se busque la integración de contenidos, estrategias de comunicación efectivas, fomento de la cognición del estudiantado y la contextualización cultural solo puede redundar en una experiencia de aprendizaje más significativo. De igual forma, el uso generalizado, por parte del profesorado, de metodologías activas y estrategias de andamiaje que permitan guiar y apoyar la adquisición y comprensión de contenidos académicos por parte del alumnado solo puede redundar en una mejora de sus experiencias formativas.

Sin embargo, esta metodología también puede presentar algunas limitaciones en su implementación, las cuales se deben mencionar. Así es destacable que se prioriza el dominio del lenguaje, el uso de la lengua materna y la lengua inglesa, en detrimento del aprendizaje de los conocimientos básicos de las áreas de conocimiento que se imparten en esas lenguas. Para poder combinar ambas finalidades es preciso el trabajo colaborativo entre el profesorado, no solo del profesorado implicado en esta docencia, sino también del conjunto de docentes del centro educativo. 
Además, la heterogeneidad del alumnado y, más concretamente, la dificultad de mantener la atención de la totalidad de estudiantes en las explicaciones y actividades de clase exige una enseñanza variada y adaptada a cada persona y ello requiere tiempo y esfuerzo. En definitiva, este enfoque demanda un profesorado comprometido y un esfuerzo compartido entre todo el equipo docente.

Para concluir, las autoras consideran que el enfoque AICLE no solo supone un reto para profesorado y alumnado, sino para el sistema educativo en general, pues ayuda a reflexionar tanto sobre la enseñanza de las lenguas como la de otras áreas curriculares. En este sentido, se propone un mayor reconocimiento del esfuerzo y compromiso docente y una formación que responda a las necesidades reales del profesorado participante, al igual que se recomienda fomentar el intercambio de experiencias intercentros a través de la creación de una cultura de coordinación y colaboración que vaya más allá de cada centro.

\section{Declaración de Material complementario}

Este artículo tiene disponible, como material complementario:

-La versión preprint del artículo en https://doi.org/10.5281/zenodo.4042072

\section{Referencias}

Aragón Méndez, M. del M. (2007). Las ciencias experimentales y la enseñanza bilingüe. Revista Eureka sobre Enseñanza y Divulgación de las Ciencias, 4(1), 152-175. https://revistas.uca.es/ index.php/eureka/article/view/3839/3416

Ardila, A. (2012). Ventajas y desventajas del bilingüismo. Forma y función, 25(2), 99-104. https:// revistas.unal.edu.co/index.php/formayfuncion/article/view/39833/41754

Bialystok, E., Barac, R., Blaye, A y Poulin-Dubois, D. (2010). Word mapping and executive functioning in young monolingual and bilingual children. Journal of Cognition and Development, 11(4), 485-508. https://doi.org/10.1080/15248372.2010.516420

Bolarín Martínez, M. J., Porto Curras, M. y García-Villalba Navaridas, R. M. (2012). Los programas bilingües en la región de Murcia: Situación y valoraciones de profesores de disciplinas no lingüísticas. Educatio Siglo XXI, 30(2), 255-288. https://revistas.um.es/educatio/article/ view/160811

Bovellan, E. (2014). Teachers'beliefs about learning and language as reflected in their views of teaching materials for content and language integrated learning (CLIL) [Tesis doctoral]. University of Jyväskylä, Finlandia. 
http://doi.org/10.15359/ree.25-2.26

http://www.una.ac.cr/educare

educare@una.ac.cr

Coyle, D. (2002). Relevance of CLIL to the European Commission's language learning objectives. En D. Marsh (Ed), CLIL/EMILE the european dimension: Actions, trends and foresight potential (pp. 27-28). University of Jyväskylä. https://jyx.jyu.fi/handle/123456789/47616

Coyle, D., Hood, P. y Marsh, D. (2010). CLIL: Content and language integrated learning. Cambridge University Press.

Cummins, J. (2002). Lenguaje, poder y pedagogía: Niños y niñas bilingües entre dos fuegos. Morata.

Custodio Espinar, M. (2019). Los retos del docente AICLE. Padres y Maestros, 378, 24-30. https:// doi.org/10.14422/pym.i378.y2019.004

Dalton-Puffer, C. (2008). Outcomes and processes in content and language integrated learning (CLIL):Current research from Europe. En W. Delanoy y L.Volkmann (Eds.), Futureperspectives for english language teaching (pp 139-157). Carl Winter. https://doi.org/10.1075/Illt.20

de Matos, M. E. E. (2014). CLIL as a catalyst for developing reflective practice in foreign language teacher education [Tesis doctoral]. Universidad de Porto, Portugal. https://repositorioaberto.up.pt/handle/10216/78036

Delmastro, A. L. (2008). El andamiaje docente en el desarrollo de la lectura y la escritura en lengua extranjera. Paradigma, 29(1), 197-230. http://www.scielo.org.ve/scielo.php?script=sci artt ext\&pid=S1011-22512008000100011

Dobson, A., Pérez Murillo, M. D. y Johnstone, R. (2011). Programa de Educación Bilingüe en España. Informe de evaluación. Resultados de la investigación independiente sobre el Programa de Educación Bilingüe del Ministerio de Educación y el British Council en España. Ministerio de Educación; British Council. https://redined.mecd.gob.es/xmlui/ handle/11162/66486?locale-attribute $=$ gl

Döpke, S. (Ed.). (2003). Australian Newsletter for Bilingual Families, O(0) 1-5. http://www. bilingualoptions.com.au/consANBF0 0.pdf

EURYDICE y Comisión Europea. (2006). Aprendizaje integrado de contenidos y lenguas (AICLE) en el contexto escolar europeo. Ministerio de Educación y Ciencia, Secretaría General de Educación.

Frigols Martín, M. J.y Marsh, D. (2014). Informe de evaluación externa. Programa Clil de la Consejería de Educación, Universidades y Sostenibilidad de Canarias. Gobierno de Canaras, Consejería de Educación, Universidades y Sostenibilidad. https://consejoescolardecanarias.org/ informe-de-evaluacion-externa-programa-clil/ 
http://doi.org/10.15359/ree.25-2.26

Guazzieri, A.V. (2008). Oral interaction in CLIL student-led cooperative group work. En M. Coonan (Ed.), CLIL e l'appredimento delle lingue. Le sfide del nuovo ambiente di apprendimento (pp. 79-103). University Ca' Foscari.

Halbach, A. (2015). Una reflexión oportuna: La formación inicial del profesorado en CLIL. En Levy M. (Head). Formación inicial para profesores de programas bilingües en inglés: Políticas, prácticas y recomendaciones (pp.17-20). Universidad de Alcalá. https://www.researchgate. net/publication/301587806 La Formacion del Profesorado para la Ensenanza Bilingue Pasado Presente_y Futuro

Harjanne, P. y Tella, S. (2008). Strong signals in foreign language education, with a view to future visions. En S. Tella (Ed.), From brawn to brain: Strong signals in foreign language education [proceedings of the ViKiPeda-2007. Conference in Helsinki Research report 290] (pp. 5584). University of Helsinki. https://www.researchgate.net/publication/339003196_From Brawn to Brain Strong Signals in Foreign Language Education

Kiely, R. (2011). Understanding CLIL as an innovation. Studies in Second Language Learning and Teaching, 1(1), 153-171. https://doi.org/10.14746/ssllt.2011.1.1.9

Lázaro, A. y García Mayo, M. del P. (2012). L1 use and morphosyntactic development in the oral production of EFL learners in a CLIL context. International Review of Applied Linguistics, 50, 135-160. https://doi.org/10.1515/iral-2012-0006

Lorenzo, F., Trujillo, F. y Vez, J. M. (2011) Educación bilingüe: Integración de contenidos y segundas lenguas. Síntesis. https://www.sintesis.com/educacion-10/educacion-bilingueelibro-1604.html

Maljers, A., Marsh, D. y Wolff, D. (Eds.) (2007). Windows on CLIL. Content and language integrated learning in the spotlight. European Platform for Dutch Education.

Marsh, D., Mehisto, P., Wolff, D. y Frigols Martin, M. J. (2010). European framework for CLIL teacher education: A framework for the professional development of CLIL teachers (pp. 10-18) European Centre for Modern Languages.

Massler, U., loannou-Georgiou, S. y Steiert, C. (2011). Effective CLIL teaching techniques. En S. loannou-Georgiou y P. Pavlou. (Eds.), Guidelines for CLIL implementation in primary and preprimary education (pp. 66-97). European Commission.

Méndez García, M. del C. y Pavón Vázquez, V. (2012). Investigating the coexistence of the mother tongue and the foreign language through teacher collaboration in CLIL contexts: Perceptions and practice of the teachers involved in the plurilingual programme in Andalusia. International Journal of Bilingual Education and Bilingualism, 15(5), 573-592. https://doi.org/10.1080/13670050.2012.670195 
http://doi.org/10.15359/ree.25-2.26

http://www.una.ac.cr/educare

educare@una.ac.cr

Pastor Martínez, M. R. (2011). CLIL and cooperative learning. Encuentro, 20, 109-118. http:// www3.uah.es/encuentrojournal/index.php/encuentro/issue/viewlssue/11/20

Pavón Vázquez, V. y Ellison, M. (2013). Examining teacher roles and competences in content and language integrated learning (CLIL). Lingvarvm Arena, 4, 65-78. https:// pdfs.semanticscholar.org/59c7/57b80db2c9ca320faa820b363ba592bd07b8.pdf? $\mathrm{ga}=2.155536376 .2142822189 .1589626381-1396058422.1589626381$

Pavón Vázquez, V.y Rubio, F. (2010). Teachers' concerns and uuncertainties about the introduction of CLIL programmes. Porta Linguarum, 14, 45-58. https://www.ugr.es/ portalin/ articulos/PL numero14/3\%20Teachers\%20Concerns\%20and\%20Uncertainties V\%20 Pavon F\%20Rubio.pdf

Pérez Márquez, M. E. (2008). La enseñanza del inglés: Un antes y un después de la implantación de la enseñanza bilingüe. Aula de Innovación Educativa, 168, 17-20.

Pérez Vidal, C. (2008). El enfoque integrado de contenidos y lenguas en Europa: Principios sociales educativos y psicolingüísticos. Aula de Innovación Educativa, 168, 7-16.

Ramos, A.M. (2011). The cultural knowledge of monolingual and bilingual student. En D. Madrid y S. Hughes (eds.). Studies in bilingual education (pp. 223-228). Frankfurtam-Main: Peter Lang.

Roza González, M. B. (2005). El marco común europeo de referencia de las lenguasy su implicación en el ámbito escolar. http://www.quadernsdigitals.net/index.php?accionMenu=hemeroteca. VisualizaArticulolU.visualiza\&articulo id=8649

Signoret Dorcasberro, A. (2003). Bilingüismo y cognición: ¿Cuándo iniciar el bilingüismo en el aula? Perfiles Educativos, 25(102), 6-21. http://www.scielo.org.mx/pdf/peredu/v25n102/ v25n102a2.pdf

Wolff, D. (2012). The european framework for CLIL teacher education. Synergies, 8, 105-116. https://gerflint.fr/Base/Italie8/dieter wolff.pdf 